\section{THE VALUE OF TEMPORARY COLOSTOMY *}

\section{LOUIS J. HIRSCHMAN, M.D. DETROIT}

Diseased or injured limbs and joints respond to treatment most effectively when function is suspended and immobilization is effected. Physiologic rest alone is a valuable therapeutic agent because the organ involved can, so to speak, devote its entire energy to the business of getting well. Unfortunately, many organs, which would be benefited by the complete suspension of activity, are so vital to life that complete rest is an impossibility. "A half a loaf is better than no bread." When one cannot secure complete cessation of functional activity, a minimum of function must suffice. Fortunately, the colon can be completely excluded from functional activity, and in the treatment of various pathologic conditions which affect it, relief and cure can often be effected by a temporary interference with its activity.

Enterostomy and colostomy are both being utilized with increasing frequency for temporary purposes. The number of lives saved yearly by the employment of temporary enterostomy in cases of postoperative ileus must run into considerable figures. The prolongation of life and increased comfort in incurable conditions of the lower bowel, and complete relief from many infective diseases of the colon made possible only by temporary colostomy, is evidenced by its increasing employment in recent years. While colostomy is a surgical procedure, perhaps its employment in the treatment of colonic disease offers as much promise to the internist and the gastro-enterologist, in the successful treatment of disease of the large intestine, as to the proctologist.

All forms of chronic colitis, whether the infective agent be bacterial or amebaid, or both, are characterized by two principal symptoms. These two symptoms are evidence of the attempt on the part of the organism to rid itself of irritating material, and accompany practically all diseases of the colon. These are evidence of increased physiologic activity of the colon, and are (1) hyperperistalsis and (2) hypersecretion.

Anything which irritates the colon, whether it be acrid and undigested food material, the inflammation produced by bacterial or ameboid activity, or the ulceration or deformity which come in the later stages of this type of colonic disease, will call on this organ for increased peristaltic activity in the endeavor to expel the undesirable occupant. In chronic disease, an atonic condition will supervene; the bowel musculature loses its tone; destructive processes extend with greater freedom; permanent loss of function, chronic invalidism, and ultimately death follow as a natural sequence unless prompt and efficient remedial measures are undertaken. It makes no difference whether the infection is syphilitic, tuberculous or dysenteric, the result of metallic or food poisoning, or the simple ulcerative type; suspension of physiologic activity, in addition to the administration of specific measures or remedies by hypodermic, internal administration or local application is of prime importance.

* Read before the Section on Gastro-Enterology and Proctology at the Seventy-Third Annual Session of the American Medical Association, the Seventy-Third Ar, May, 1922.
Patients of another large class, those suffering from the later manifestations of specific diseases, strictures, complicated fistulas, and neoplasms of either benign or malignant nature, are often converted from incurable to curable by the employment of temporary colostomy before the indicated major surgery is carried out. Temporary colostomy is also of distinct value in the treatment of malignant growths, which appear inoperable, and when radium or deep high-voltage roentgen-ray therapy is employed. Not only is the temporary suspension of physiologic activity of great assistance in the treatment of diseases of the colon, distal to the colostomy, but this artificial opening offers an opportunity to irrigate the diseased organ from above downward.

The administration of colonic irrigations by means of the rectal tube is merely of fractional value. The solution, passing up into and through the diseased portion of the rectum and colon, carries with it the products of disease. On the return flow and expulsion of the solution, much of this material remains in the cecum and along the walls of the colon, and continues to infect, irritate and inflame its walls.

A great step in advance was made in colonic irrigation when appendicostomy and cecostomy were first performed, and irrigation from above downward was successfully accomplished. Partial physiologic rest was thus obtained, and a much more thorough cleansing of the diseased bowel was procured. While the feces were not prevented from passing through the diseased portion of the canal, they were so diluted by the irrigating solution that they were not nearly so irritating. Many types of colitis were entirely cured by this method when combined with the indicated medicinal and dietetic therapy. When the patient, however, is rapidly losing ground and becoming anemic from the continual loss of blood from diseases such as chronic ulcerative colitis, colonic polyposis and amebic dysentery, on the one hand, and from the systemic effect of infective and suppurative conditions, such as ulceration combined with stricture, multiple fistulas of the lower colon communicating with other organs, such as the bladder, vagina, tubes or appendix, or multiple diverticulitis, the organ must be given as near absolute rest as possible. This can be accomplished only by enterostomy or colostomy well above the diseased area.

The point of election for the artificial anus can be determined only after a thorough roentgenologic study of the gastro-intestinal tract, corroborated by surgical examination through a laparotomy wound. All things being equal, an abdominal incision, through the rectus muscle on either side of the median line, should be made for exploratory purposes, and either this or, more rarely, a second wound should be used for the colostomy.

The question of anesthesia for the performance of temporary colostomy is an important one. Patients suffering from chronic diseases of the colon are not, as a rule, ideal patients for abdominal surgery. They are usually debilitated and suffering from disturbed nutrition, and their resistance is markedly subnormal. They are particularly prone to complications resulting from anesthetic accidents when ether is employed. If, in the surgeon's judgment, complete surgical narcosis is deemed absolutely necessary, the use of gas and oxygen combined with local anesthesia is much to bo preferred to ether. In my practice I find it rarely necessary to employ general anesthesia for the per- 
formance of temporary colostomy. My preference is for the use of local regional anesthesia produced by the infiltration of 0.5 per cent. solutions of either apothesin or procain. This is not combined with epinephrin, as I find no reason to add this drug to secure perfect anesthesia.

One hour before the time selected for operation, the patient is given from a quarter to a third of a grain of morphin sulphate, combined with from $1 / 150$ to $1 / 100$ grain of scopolamin hydrobromid. The administration of this combination induces a state of mental calm and dispels the anticipatory anxiety and dread of a surgical operation, and assists in no small degree the relaxation of the parts produced by local anesthesia. The quantity of anesthetic solution is of relative unimportance. The only criterion used is infiltration sufficient to produce complete nerve block. No solution is injected into or between the layers of the skin, but the needle is introduced underneath the skin so that we have a true subcutaneous and not a subcuticular or intradermal infiltration. It is the latter type of injection, and the use of epinephrin, which is responsible for the skin slough, which has been unjustly attributed to anesthetic agents.

An area from 1 to 2 inches wide and 6 inches long is infiltrated down to the fascia. This, in turn, is punctured and infiltrated for the same length. Then after from three to five minutes, all tissues are divided down through the fascia. The underlying muscles are then thoroughly distended with anesthetic solution, and after a short pause the fibers are incised or separated by blunt dissection and the peritoneum is exposed. The hypodermic needle is inserted between the layers of the peritoneum, and the line of proposed incision is infiltrated. The peritoneum is opened in the same manner as under general anesthesia, and retracted. The assistants must bear in mind at all times that the patient is not asleep or under general anesthesia, and all unnecessary conversation, noises and handling of tissue are strictly enjoined. Unnecessary trauma to tissues is avoided under local anesthesia, and the patient is thereby the gainer.

Examination of the abdominal viscera can now be made. The colonic lesion is then located, and the portion of the colon well above the diseased segment is delivered outside the wound. If there is any evidence of pain on handling the colon, the mesentery below the part grasped should be infiltrated with the anesthetic solution, after which it will be found that it can be freely and painlessly manipulated. The mesentery is perforated with blunt forceps, and a thick-walled rubber tube, not larger than a lead pencil, is drawn through to act as a support for the bowel on the abdominal surface. It is not necessary or advisable to suture the skin, fascia or peritoneum to the bowel, as adhesions will form within a few hours between the cutaneous abdominal surfaces and the bowel wall.

It is my practice to place the exposed loop or colon as high in the wound as possible, so that it may be well out of the way in the event of further abdominal surgery for the removal of colonic disease in the pelvis, colon or rectum. The abdominal wound below the colonic loop is sewed in layers, and the skin closed with clips. The parts are dusted with zinc stearate powder, pads are applied under the ends of the supporting rubber tube, a dressing is applied, and the patient is returned to bed until the bowel is opened forty-eight hotirs later.
The whole operation is performed in from ten to fifteen minutes, and the patient is able to continue his nourishment without interruption. There is practically no postoperative pain, and the patient shows no reaction and gains several days over the one who had the same operation performed under general anesthesia. If, at any time during the operation, conditions arise which prevent its completion under local anesthesia, a short period of gas anesthesia will help out. This occurs, however, in only a small percentage of cases.

If one wishes to divide the spur of the colostomy by pressure necrosis at the time of bowel closure, then it is necessary to sew the two loops of the bowel together at their mesenteric attachment for 3 or 4 inches. However, we find it unnecessary to do this, as when the time for closure arrives it may be done extraperitoneally by simple invaginating sutures under local anesthesia, or without any anesthesia whatever.

After the bowel has been closed, it is dressed with dichloramin- $T$ solution for four or five days. When healing is complete and the wound proved sterile by bacterial examination, the peritoneal cavity is opened under local anesthesia, the bowel replaced, and the wound closed by layers.

In quite a number of patients the simple suspension of peristaltic activity, through the employment of temporary colostomy, has been all the treatment required for permanent relief. In major surgical procedures on the distal portion of the bowel, such as resections, removal of neoplasms and operations for extensive fistulas, the creation of a comparatively clean field in which to work, and the physiologic rest afforded by temporary colostomy, has meant the difference between life and death.

Its more frequent employment is urged in the treatment of inflammatory and infectious diseases now treated medically as "colitis." Temporary colostomy, as a step in the surgical treatment of severe injuries, neoplasms and fistulas, is now almost universally employed in many clinics. The time for halfway medical measures, enemas and colonic irrigations, has passed, and the treatment of chronic and severe colonic diseases will be materially improved by physiologic rest and direct treatment and irrigation afforded by the more general use of temporary colostomy.

The employment of local anesthesia has robbed this operation of much of the risk inherent in etherization, has placed it among the list of safe surgical procedures, and has made it a life-saving measure of the greatest value.

\section{ABSTRACT OF DISCUSSION}

Dr. Alois B. Graham, Indianapolis: As to the value of a temporary colostomy, I agree with all that Dr. Hirschman sait. Any surgical technic or procedure which will place a pathologic area at practically complete physiologic rest, which will inhibit infection, control hemorrhage, improve appetite, add weight, and convert what was previously a relatively poor or bad surgical risk into a companatively safe surgical risk, should command the attention of all good surgeons. This is the possibility of a temporary colostomy.

Dr. Samuel G. Gant, New York: No subject in our field is of more value than temporary colostomy, although it is not new. There are many conditions in which I perform it, sometimes preceding an excision of the rectum and for polyposis; but in the majority of cases it is done for relief of inflammatory conditions in the intestines complicated by ulceration and excessive discharges. I do not think it makes much difference what organism is the cause; the inflammatory processes pave the way for mixed infection, and 
eventually there is destruction of the mucosa and surgical diarrhea. I try to classify these conditions into moderate and severe cases of colitis. If a moderate case, I treat it by rest, diet, medication and rectal irrigations. If there are quite a number of stools containing considerable blood and pus, then I employ appendicostomy or cecostomy. When the condition is an extensive mixed infective colitis, the submucosa riddled with ulcers, etc., then it is essential to put the intestine at complete rest, and in these cases I prefer a colostomy or cecostomy. However, in certain conditions we have to do as Dr. Hirschman has suggested, viz., perform colostomy instead of cecostomy. I do not believe in the treatment of these cases by ileostomy because the patient suffers from frequent fluid stools and excoriations of the skin surrounding the opening. The artificial anus must be made according to the location of the lesions. But putting the bowel at rest and employing frequent irrigation, one can cure a great many cases of chronic colitis which otherwise would end in death. The openation has a decided field, and, as a rule, can be performed under local anesthesia, for the reason that the bowel is superficial, and there is no pain in handling it when one can bring it up without much traction. Of course local anesthesia is not effective when one must pull on the mesentery. In this class of affection I think if one studies cases and employs temporary colostomy when indicated, the operation will be found serviceable. The bowel can be left open one, two or three years, and then the opening closed without atrophy or damage to the intestine. Formerly I believed that if a person did not use the bowel for a considerable time it would not function again, but such was not so in several of my cases.

Dr. Sidney K. Simon, New Orleans: It seems to me that the operation of colostomy advocated by Dr. Hirschman represents the last step in the therapy of colonic conditions. I can easily understand that from the proctologist's standpoint this measure demands more than it wotald from the purely medical side, because the proctologist sees the extreme cases, after they have passed through the hands of the medical man. I take issue with Dr. Hirschman in several respects. First of all, I object somewhat to grouping the various etiologic factors of colitis under one heading, and claiming, as he apparently does, that the treatment is surgical. Certainly, in the amebic infections of the colon there rarely arises an occasion for surgical intervention. The use of specific therapy along well known lines has in my own experience obviated the necessity for surgical intervention. In the purely bacterial infections of the colon, frequently the patient is allowed to drift because of lack of proper appreciation on the part of the attending physician of the probably serious nature of a simple colitis. The patient is treated along the usual lines with a very moderate degree of attention, and from a simple infection of the mucosa the condition pathologically is allowed to advance below the mucous membrane, affecting the musculature so that eventually the bowel becomes a fixed, nonperistaltic organ, allowing the feces as it comes through the ileocecal valve to gravitate toward the descending colon and rectum without any peristaltic control When once that condition has come about, when peristalsis is to a great extent annihilated, then and only then in my opinion should surgical intervention be resorted to. However, prior to advising surgical intervention I should employ lavage. A solution which has proved of benefit in this class of cases is methylene blue or gentian violet used as a flush of the colon from above downward. Within half an hour, after 50 c.c. has been introduced, the fluid finds it way through the colon, and one gets in effect a complete washing out of the colon from above downward which has practically the same effect as a cecostomy opening.

Dr. Alfred A. Strauss, Chicago: I am heartily in accord with the ideas of Dr. Hirschman regarding colostomy, but in the severe forms of ulcerated colitis ileostomy has many advantages over colostomy. In many of these cases the pathologic changes extend into the cecum and even into the appendix, and it is much more difficult to put the bowel at absolute rest with a colostomy than with an ileostomy. I am highly in favor of doing these operations under local anesthesia, because the patient's condition is usually very poor.
In doing an ileostomy, by making a knuckle of the ileum and then opening the ileum transversely, the entire colon will be put at absolute rest. These operations are usually done as a last resort. The patient, as a rule, has been under the care of an internist for one or two years, and since the patient is either no better or is markedly worse, surg.ty is resorted to. This is a serious mistake. The operation shumld be done early in the disease before marked patholugic changes have occurred in the colon, changes which are lound to produce cicatrization and stenosis. Besides, the 1 atient is in much better condition, and recovery is that much qu icker.

The result from ileostomy in these chronic ulcerated olitis cases is nothing short of wonderful. Patients can be treated through the ileostomy by irrigations with various solutions, which in many cases aids considerably in addition to the rest the colon gets from the ileostomy. In these severe form; of ulcerated colitis, repeated blood transfusions, one before and one after operation, are of the greatest aid to the patient, and reduce the operative risk about 50 per cent. In addition to that, for five or six days after the operation, I have given 1 quart of 5 per cent. glucose solution intravenously daily. As to the operative procedure, in spite of definite roentgenray findings, it is best always to make a small midline incision below the umbilicus to inspect the colon. Ileostomy or colostomy should be done through a separate small gridiron incision, and the midline incision closed. One of the great advantages of ileostomy over colostomy is the absence of the colon bacillus odor from the intestinal discharge.

Dr. John L. Jelks, Memphis, Tenn.: I believe that some of us are not quite clear as to the proper terminology of some of the procedures mentioned. In the first place, if I should speak of colostomy, I should mean something independent of the appendix. If I were to speak of an appendicostomy, I should refer to an operation which means picking the appendix up and using it as a nozzle through which to irrigate the colon. If I were to mention appendicocecostomy, I should mean the operation here described-that of fastening the cecum against the abdominal wall and using the opening where the appendix had been removed. Dr. Gant speaks of pain. I do not believe it is advisable to wait for that symptom, because there will be pain when the cecum is pulled up I should prefer to use a little procain previous to operation. Anticipating an operation of this type, I much prefer using morphin sulphate, three doses, before the operation is started, in order to get the patient in a fairly comfortable condition. I have seen colostomy performed to put a colon at rest which meant simply the opening of the colon and allowing the fecal material to come out. But if the colon is to be put at rest. one has to exclude; therefore one must close the distal part and set it apart from the proximal part. Dr. Simon states that this procedure is rarely indicated in amebic infection, but that well known lines of medical treatment should be followed. I believe I have seen as many amebic cases as any man in this country, and I know that when the appendix and cecum are involved, and the appendix has in it amebic cysts which one cannot get at, one is not going to cure that patient by any medical procedures whatsoever. Also, one may fail to find amebas in the stools of patients to whom emetin has been given by the colon. I never give less than 1 grain of emetin at a dose, when I give it at all. I have given bismuth in one, two or three heaping teaspoonsful doses, three or four times a day. Some men claim that they cure patients with 10 grain doses. I cannot do it. The amebas inhabit the colon, not the ileum, and there is no need of an ileostomy to cure a disease that does not involve the ileum or the small intestine. However, I agree with Dr. Simon as to the method of treating these patients by the use of various irrigants. I am especially partial to gentian violet solutions. I hope that we shall get better solutions for getting rid of these intractable infections. In transfusing, I use the citrate method.

Dr. Anthony Bassler, New York: I feel that in the strictly medical conditions of bacterial infections with ulcera. tion and infiltration in the colon, cecostomy or other surgical procedure may, in a very few instances, be required. In my experience the method mentioned by Dr. Simon has been perfectly satisfactory. Up to three years ago, the routine procedure was cecostomy and appendicostomy, with irrigations 
kept up for several months, and we had not a few patients return after a year or two with the original symptoms and often with the fistula closed, when he had to reoperate. Now practically none are operated on, and the results from medical treatment are quite as good. For irrigation $I$ use the hypertonic solutions. To these is added gentian violet, which is kept up for weeks and months. I have used other dye solutions, but the gentian violet is now used most often. During the last three years in cases of dysenteric infections, through all the grades of $B$. coli and the streptococcic infections, by persistent medical treatment only, I have achieved results which I feel are quite as good as those obtained from the operative procedures of former years; in fact, much better. When, however, it comes to malignant disease, or granulomatous diseases, such as tuberculosis and syphilis, I think that the cases must be considered surgical at the start and then perhaps medical, as Dr. Hirschman suggests.

DR. Granville S. Hanes, Louisville: I want to call attentention to one feature in the technic of doing colostomy, and especially when it is done with the view of treating a pathologic condition in the lower segment. It formerly was advised that when the sigmoid was brought up through the abdominal wound, the bowel should be pulled down and made tight from above, leaving the redundant part below. Just the converse should be done. The redundant part of the sigmoid should be left above the colostomy opening in order that it may serve as a reservoir for the material as it comes down, with the result that in many cases the patient has a movement every morning just as before there were any pathologic changes. Then, especially in cases of carcinoma, if necessary to introduce radium, this becomes entirely possible, while with the redundant part of the intestine below the colostomy it is impossible to introduce it. Therefore, not alone from the standpoint of introducing an instrument into the lower segment of the intestine for the purpose of making local applications of any kind or applying radium, it also serves as a reservoir for fecal material. Again, I would call attention to the fact that it is possible to do colostomy easily in only two portions of the intestine. These are the transverse portion of the colon and the sigmoid. Other portions of the large bowel, except the cecum, usually have mesenteric attachments so short that they cannot be delivered through the abdominal incision.

Dr. L. J. Hirschman, Detroit: I merely offer the operation of temporary colostomy to those who are surgeons but who are treating cases medically, as a method of very decided assistance to them in shortening the period of treatment. In the treatment of infective conditions of the colon, a great deal of time can be saved to the patient with the performance of a colostomy and its closure under local anesthesia. It is one of the most conservative operations one could advise. Every surgeon has found that a temporary colostomy often gives permanent relief in a condition which he thought would require more radical measures. In regard to the question of using this method to assist in the treatment of amebic dysentery, Dr. Jelks has answered that. I have found it of service in late cases of dysentery. With reference to the location of colostomy, we must be guided by the lesion in the individual case as to whether we shall do an ileostomy, a high or low colostomy, or what portion of the intestine shall be used. My experience shows that most of these conditions do not involve the entire colon, and many times we have been able to select the part that Dr. Hanes has mentioned, the transverse and sigmoidal portions. In many cases in which I previously would have thought ileostomy was necessary, I have found that colostomy answered the purpose. Dr. Hanes has mentioned the question of drawing the upper segment of the bowel taut. This depends on conditions. If one wants to pull up the intestine in order to form a reservoir, well and good. But if later one has to excise a carcinoma or other lesion, then it is well to leave as much intestine below the colostomy as possible in order to have good material with which to make an anastomosis. So far as impeding peristalsis is concerned, my experience has been exactly the opposite. I have found that, with convalescence, peristalsis is increased if anything.

\section{AN ANALYSIS OF FOURTEEN CON- SECUTIVE CASES OF SPINAL CORD TUMOR *}

\author{
CHARLES H. FRAZIER, M.D. \\ AND \\ WILLIAM G. SPILLER, M.D. \\ PHILADELPHIA
}

We present this critical analysis of a series of fourteen consecutive cases in the hope that the emphasis which we lay on certain diagnostic phases may lead to an earlier diagnosis in spinal cord tumors and, as a result, to the consideration of surgical therapy before cord degeneration - the inevitable consequence of delay - has deprived the patient of relief from crippling deformities. Whatever the cause, the fact remains that in this series the final diagnosis was not established until an average of two and a half years had elapsed from the date of the initial symptom.

While tumors of the spinal cord are less frequent than brain tumors in the ratio of one to six, the proportion of operable tumors of the cord from the standpoint of localization and feasibility of exposure and

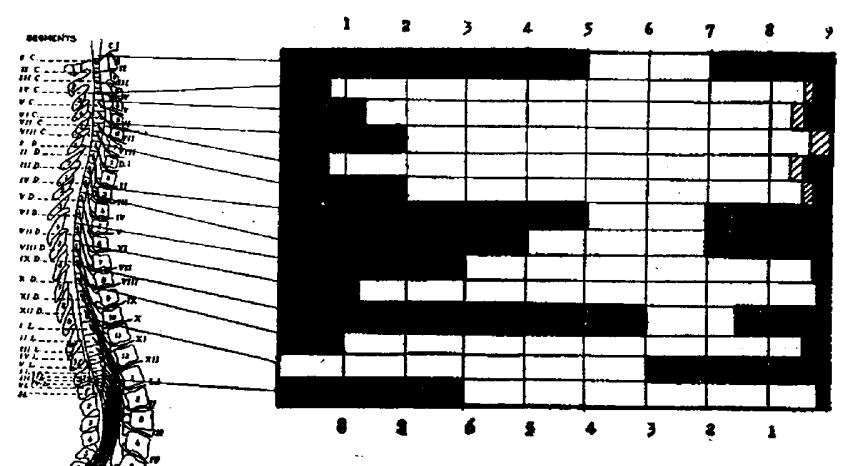

Fig. 1.-Duration of pain in years (at left) as compared with the duration of motor impairment in the individual tumor cases of this series (at right). Dark areas at left represent interval of time from beginning of pain. Dark areas at right show dura
weakness of legs; shaded areas, weakness and atrophy of arms.

removal is far greater. In twelve of our fourteen cases the tumor was accurately localized, accessible, well encapsulated and distinctly an operable lesion.

\section{DIAGNOSIS}

Fully cognizant of the fact that occasionally spinal cord tumors run a painless course, in this series in thirteen out of fourteen cases not only was pain a conspicuous symptom, but in each of these thirteen cases it was the first symptom. There are other important lessons to be learned from this pain phenomenon. While in later stages pain may be referred to a more widespread distribution, the original pain zone continues throughout the course of the disease and is therefore an important localizing sign when the time comes to determine the seat of the growth. The physician might be misled by the occasional remission, as often happens, and interpret these remissions as responses to the treatment he may have inaugurated.

Of interest, too, is the length of time which elapses between the onset of pain and the first signs of motor impairment. In four, or one third of the cases, three

* Read before the Section on Nervous and Mental Diseases at the Seventy-Third Annual Session of the American Medical Association, 